\title{
Swelling of mucoadhesive electrospun chitosan/polyethylene oxide nanofibers facilitates adhesion to the sublingual mucosa
}

Stie, Mai Bay; Gätke, Johan Ring; Wan, Feng; Chronakis, loannis S.; Jacobsen, Jette; Nielsen, Hanne Mørck

\section{Published in:}

Carbohydrate Polymers

Link to article, DOI:

10.1016/j.carbpol.2020.116428

Publication date:

2020

Document Version

Peer reviewed version

Link back to DTU Orbit

Citation (APA):

Stie, M. B., Gätke, J. R., Wan, F., Chronakis, I. S., Jacobsen, J., \& Nielsen, H. M. (2020). Swelling of mucoadhesive electrospun chitosan/polyethylene oxide nanofibers facilitates adhesion to the sublingual mucosa. Carbohydrate Polymers, 242, [116428]. https://doi.org/10.1016/j.carbpol.2020.116428

\section{General rights}

Copyright and moral rights for the publications made accessible in the public portal are retained by the authors and/or other copyright owners and it is a condition of accessing publications that users recognise and abide by the legal requirements associated with these rights.

- Users may download and print one copy of any publication from the public portal for the purpose of private study or research.

- You may not further distribute the material or use it for any profit-making activity or commercial gain

- You may freely distribute the URL identifying the publication in the public portal 


\section{Journal Pre-proof}

Swelling of mucoadhesive electrospun chitosan/polyethylene oxide nanofibers facilitates adhesion to the sublingual mucosa

Mai Bay Stie (Conceptualization) (Methodology) (Formal analysis) (Investigation) (Writing - original draft) (Visualization) (Project

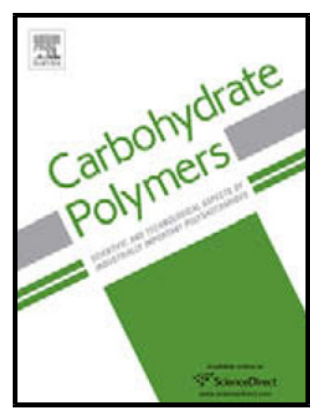
administration), Johan Ring Gätke (Methodology) (Investigation) (Writing - review and editing), Feng Wan (Methodology) (Investigation) (Writing - review and editing), loannis S. Chronakis (Conceptualization) (Validation) (Writing - review and editing) (Supervision), Jette Jacobsen (Conceptualization) (Validation) (Writing - review and editing) (Supervision), Hanne Mørck Nielsen (Conceptualization) (Validation) (Writing - review and editing) (Supervision) (Funding acquisition)

PII: S0144-8617(20)30602-0

DOI: https://doi.org/10.1016/j.carbpol.2020.116428

Reference:

CARP 116428

To appear in:

Carbohydrate Polymers

Received Date: $\quad 10$ April 2020

Revised Date: 4 May 2020

Accepted Date: $\quad 5$ May 2020

Please cite this article as: Stie MB, Gätke JR, Wan F, Chronakis IS, Jacobsen J, Nielsen HM, Swelling of mucoadhesive electrospun chitosan/polyethylene oxide nanofibers facilitates adhesion to the sublingual mucosa, Carbohydrate Polymers (2020), doi: https://doi.org/10.1016/j.carbpol.2020.116428 
This is a PDF file of an article that has undergone enhancements after acceptance, such as the addition of a cover page and metadata, and formatting for readability, but it is not yet the definitive version of record. This version will undergo additional copyediting, typesetting and review before it is published in its final form, but we are providing this version to give early visibility of the article. Please note that, during the production process, errors may be discovered which could affect the content, and all legal disclaimers that apply to the journal pertain.

(C) 2020 Published by Elsevier. 


\title{
Swelling of mucoadhesive electrospun chitosan/polyethylene oxide nanofibers facilitates adhesion to the sublingual mucosa
}

\author{
Mai Bay Stie ${ }^{1,2}$, Johan Ring Gätke ${ }^{1,2}$, Feng Wan ${ }^{1,2}$, Ioannis S. Chronakis ${ }^{3}$, Jette Jacobsen ${ }^{1}$ and \\ Hanne Mørck Nielsen ${ }^{1,2, *}$ \\ ${ }^{1}$ Department of Pharmacy, University of Copenhagen, Universitetsparken 2, 2100 Copenhagen, Denmark
${ }^{2}$ Center for Biopharmaceuticals and Biobarriers in Drug Delivery, Department of Pharmacy, University of Copenhagen,
Universitetsparken 2, 2100 Copenhagen, Denmark
${ }^{3}$ Nano-BioScience Research Group, DTU-Food, Technical University of Denmark, Kemitorvet, B202, 2800 Kgs. Lyngby, Denmark
}

*e-mail: hanne.morck@sund.ku.dk, tel.no.: +45 35336346

\section{Highlights}

- Stability of chitosan/PEO nanofibers in water depends on the DDA of chitosan.

- Chitosan interacts strongly with mucin from bovine submaxillary glands.

- Chitosan with the highest DDA (96\%) interacts most strongly with mucin.

- Swelling of chitosan/PEO nanofibers induces adhesion to sublingual mucosa.

\begin{abstract}
Mucoadhesive chitosan-based electrospun nanofibers are promising candidates for overcoming challenges associated with sublingual drug delivery, yet studies focusing on evaluating the mucoadhesive properties of nanofibers for sublingual administration are limited. The aim was to elucidate the mucoadhesive properties of chitosan/polyethylene oxide (PEO) nanofibers focusing on how the degree of deacetylation (DDA, 5396\%) of chitosan influenced their morphological and mucoadhesive properties. The mechanism of mucoadhesion was explained by the intermolecular interactions of chitosan with mucin from bovine submaxillary glands using quartz-crystal microbalance with dissipation monitoring and by adhesion of the nanofibers to ex vivo porcine sublingual mucosa. An increase in chitosan DDA improved the morphological stability of the nanofibers in water, but did not contribute to altered mucoadhesive properties. This study demonstrates excellent mucoadhesive properties of chitosan/PEO nanofibers and shows that the strong mucoadhesiveness of the nanofibers is attributed to their swelling ability.
\end{abstract}

Keywords: Mucoadhesion, electrospun nanofibers, chitosan, sublingual drug delivery 


\section{Introduction}

The mucosa in the oral cavity is an attractive site for drug delivery of highly gastro-intestinal or hepatic first passage metabolized compounds as it is easily accessible, can be applied by non-invasive means and the tissue underlying the epithelium is highly vascularized with blood supply circumventing the liver. The epithelium on the ventral side of the tongue is only 8-12 cell layers in thickness and non-keratinized, which makes the sublingual tissue significantly more permeable than e.g. the buccal or gingival mucosa (Kraan et al., 2014; Squier \& Kremer, 2001; Teubl et al., 2013) and thus a relevant site for absorption of poorly permeable drug molecules. However, the sublingual epithelium is still a significant barrier for drug absorption (Rathbone, Drummond, \& Tucker, 1994). In addition, sublingual drug delivery is challenged by a continuous flow of saliva, which leads to loss of drug by washout and swallowing (Rathbone et al., 1994).

Drug permeation is highly dependent on the residence time and the concentration gradient of drug across the epithelium (Khutoryanskiy, 2011). Mucoadhesive drug delivery systems that facilitate release of drug for a prolonged period of time at the site of application, and thereby maintain a high drug concentration gradient across the tissue, will improve the total drug permeation through the mucosa. Encapsulation of drugs in innovative drug delivery systems may also further protect the drug from degradation and washout by saliva, as well as control the drug release kinetics. With the right design, improvement of systemic bioavailability of the drug and, importantly, patient convenience can be achieved by sublingual administration compared to injection of e.g. peptide drugs. Furthermore, for local therapy in the oral cavity, mucoadhesion can increase retention at the application site, potentially result in a lower required total dose for site-specific delivery, and reduce side effects caused by drug exposure to off-target sites such as the gastro-intestinal tract upon swallowing. 
Electrospun nanofibers have a high surface-area-to-volume ratio, tunable surface structures, facilitate controlled drug release and provide great prospects for industrial scale up (Chronakis, 2010). Nanofibers thus provide an opportunity for overcoming the challenges associated with sublingual drug delivery and can potentially improve the delivery of not only small molecules, but also biopharmaceuticals such as peptides via the sublingual route. Nanofibers have been explored for oromucosal drug delivery of amongst others small molecule drugs for local anesthesia (Clitherow et al., 2019), nanoparticles for vaccination (Mašek et al., 2017), as well as insulin for transbuccal delivery (Lancina, Shankar, \& Yang, 2017). However, research has rarely focused on evaluating and improving the mucoadhesive properties of electrospun nanofibers for sublingual drug delivery.

Chitosan is a biopolymer derived from deacetylation of chitin and consists of N-acetyl-D-glucosamine and D-glucosamine. The degree of deacetylation (DDA) is highly important for the physicochemical properties of chitosan and affects the biological performance of the polymer. Amongst a plethora of favorable biomedical properties, chitosan is well known to be hygroscopic with good swelling abilities, and the polymer is therefore extensively explored for its excellent mucoadhesive properties. Furthermore, the amino groups of chitosan are protonated under acidic conditions and interacts strongly with the negatively charged mucins expressed on epithelial mucosal surfaces and in saliva (Ways, Lau, \& Khutoryanskiy, 2018). The number of amino groups of chitosan and thus the cationic charge density of chitosan is dependent on the DDA of the polymer. Various mucoadhesive drug delivery systems based on chitosan have been reported such as nanoparticles, hydrogels and also nanofibers intended for mucosal drug delivery (Bernkop-Schnürch \& Dünnhaupt, 2012; Lancina et al., 2017). Electrospinning of neat chitosan has previously only been achieved in highly acidic dispersion such as 90\% acetic acid (Geng, Kwon, \& Jang, 2005), in trifluoroacetic acid (TFA) and in combination with volatile organic solvents such as dichloromethane (Ohkawa, Cha, Kim, Nishida, \& Yamamoto, 2004). As recently demonstrated and as a more patient-friendly alternative, chitosan- 
based nanofibers can be electrospun under safe conditions applying only biocompatible excipients 'generally recognized as safe' by the US Food and Drug Administration (FDA) by including a watersoluble co-spinning polymer such as polyethylene oxide (PEO) and dilute aqueous acidic acid as a solvent (Duan, Dong, Yuan, \& Yao, 2004; Stie et al., 2019).

In this study, chitosan/PEO nanofibers containing chitosan of various DDA (53-96\%) were produced by electrospinning, their morphology characterized by scanning electron microscopy (SEM) and the composition confirmed by Fourier-transform infrared spectroscopy with attenuated total reflection (FTIR ATR). The objective of this study was to explore the mucoadhesive properties of electrospun chitosan/PEO nanofibers specifically for sublingual administration. We hypothesized that the properties of the electrospun chitosan-based nanofibers, e.g. the morphological stability in water, depend on the DDA of chitosan. Also, it was hypothesized that the DDA of chitosan affects the interaction of the polymer with mucin derived from bovine submaxillary glands due to a higher cationic charge density of chitosan. A systematic and comparative study was conducted to investigate the biophysical interaction of chitosan with an immobilized mucin layer by quartz crystal microbalance with dissipation monitoring (QCM-D). We demonstrate that chitosan/PEO nanofibers possess strong adhesion to porcine sublingual mucosa ex vivo by measuring the work of adhesion and that the mucoadhesive properties mainly relates to extraordinary swelling properties of the chitosan/PEO nanofibers. 


\section{Materials and methods}

\subsection{Materials}

Chitoceuticals chitosan 70/100 (DDA 71\%, Mw 100-250 kDa, chitosan-71), chitosan 80/100 (DDA 82\%, Mw 100-250 kDa, chitosan-82), and chitosan 95/100 (DDA 96\%, Mw 100-250 kDa, chitosan96) was purchased from Heppe Medical Chitosan (Halle, Germany); According to the manufacturer, all chitosans were produced from raw materials of pharmaceutical quality grade. Chitosan was acetylated from chitosan 95/100 to obtain chitosan-53 (DDA 53\%, Mw-100-250 kDa, chitosan-53, purity >97\%) (Supporting Information). PEO (Mw $\sim 900 \mathrm{kDa}$, contained $\leq 1 \%$ alkalies and other metals), mucin from bovine submaxillary glands type I-S, acetic acid anhydride (Ph.Eur. $\geq 99 \%$ ), pyridine ( $\geq 99 \%$ ), Hank's balanced salt solution (HBSS), Dulbecco's phosphate buffered saline (PBS), Dulbecco's modified Eagle's medium (DMEM), glutamine, penicillin, streptomycin, phenazine methosulfate (PMS), glycerol ( $\geq 99.5 \%$ ), tributyl O-acetylcitrate (98\%), ethyl cellulose (48-49.5\% (w/w) ethoxyl basis), N-(3-dimethylaminopropyl)-N'-ethylcarbodiimide hydrochloride (EDC), N-hydroxysuccinimide (NHS), ethanolamine hydrochloride (ETA-HCl) and 11mercaptoundecanoicacid (MUA) were obtained from Sigma Aldrich (St. Louis, MO, USA). Fetal bovine serum (FBS) was purchased from PAA laboratories (Brøndby, Denmark). N-2hydroxyethylpiperazine-N'-2-ethanesulfonic acid (HEPES) was from PanReac AppliChem (Damstadt, Germany). 3-(4,5-dimethylthiazol-2-yl)-5-(3-carboxymethoxyphenyl)-2-(4sulfophenyl)-2H-tetrazolium) (MTS) was obtained from Promega (Madison, WI, USA). Acetic acid (glacial, 100\%), acetone (HPLC grade, $\geq 99.9 \%$ ) and ethanol (96\%) were purchased from VWR Chemicals BDH (Søborg, Denmark). The human TR146 cell line was obtained from European Collection of Authenticated Cell Cultures (ECACC) (Public Health England, Porton, UK). 


\subsection{Production and characterization of electrospun chitosan/PEO nanofibers}

The nanofibers were produced according to Stie et al., (2019) with small modifications. In short, dispersions of chitosan with various DDA and PEO were prepared in ultrapure water $(18.2 \mathrm{M} \Omega \times \mathrm{cm}$, purified by a PURELAB flex 4, ELGA, High Wycombe, UK) at concentrations of $2 \%(w / w)$ and $4 \%$ (w/w), respectively. The chitosans were dispersed in ultrapure water with $0.7 \%(\mathrm{w} / \mathrm{w})$ acetic acid. Both dispersions were stirred for two days at room temperature (RT) to ensure complete hydration of the polymers prior to electrospinning. Chitosan and PEO dispersions were mixed for 30 min to obtain a $1: 1(w / w)$ ratio of the polymers before electrospinning. Nanofibers were produced by electrospinning on an ES50P-10W high voltage source applying $20 \mathrm{kV}$ at $21-25{ }^{\circ} \mathrm{C}$ and at low humidity ( $<25 \%$ relative humidity) from a $1 \mathrm{~mL}$ syringe with a $20 \mathrm{G}$ blunt needle (Photo-Advantage, Ancaster, ON, Canada). The nanofibers were electrospun for $3 \mathrm{hrs}$ and collected on aluminum foil on a stainless steel collector plate placed $15 \mathrm{~cm}$ from the needle tip.

Visualization of the morphology of the nanofibers by SEM and composition analysis conducted by FTIR of the nanofibers was carried out as previously described by Stie et al., (2019). The nanofibers were visualized under a Phenome Pro X scanning electron microscope (Phenomworld, Eindhoven, The Netherlands). The diameters of the nanofibers were measured by ImageJ version $1.51 \mathrm{j} 8$ software (National Institute of Health, Bethesda, MD, USA). FTIR was performed on a Bomem MB-Series spectrophotometer (Bomem, Québec, QC, Canada) with an Arid-Zone ${ }^{\mathrm{TM}}$ light source spectrometer based on a universal FTIR sensor equipped with DuraScope diamond ATR (SensIR Technologies, Danbury, CT, USA). 


\subsection{Preparation of hydrophobic film as non-mucoadhesive control}

A hydrophobic film, a non-mucoadhesive control sample, was produced by dispersing $141 \mathrm{mg}$ acetyl tributyl citrate, $47 \mathrm{mg}$ glycerol, and $750 \mathrm{mg}$ ethyl cellulose in $15 \mathrm{~mL}$ acetone. After stirring for at least 3 hrs at RT, $10 \mathrm{~mL}$ was left to dry at RT in a Petri dish.

\subsection{Weight loss of nanofibers after exposure to water}

The weight loss was determined for $2 \mathrm{mg}( \pm 10 \%)$ nanofibers and for discs of $10 \mathrm{~mm}$ in diameter of the hydrophobic film ( $\left.\mathrm{m}_{\text {initial }}\right)$ placed in metal baskets submerged in $3 \mathrm{~mL}$ warm $\left(37^{\circ} \mathrm{C}\right)$ ultrapure water and incubated for $3 \mathrm{hrs}$ at $37^{\circ} \mathrm{C}$ with mild shaking (50 rpm) on a shaking table (Thermo MaxQ 2000, Thermo Fisher Scientific, West Palm Beach, FL, USA) with a custom-built chamber connected to a water bath for temperature control. The baskets were drawn from the water and gently blotted on filter paper to remove water, and the fibers were left to dry until constant weight $\left(\mathrm{m}_{\text {final }}\right)$ at $\mathrm{RT}$ in a fume hood. The weight loss was determined (Eq. 1).

$$
\text { Weight loss }(\%)=\frac{m_{\text {intial }}-m_{\text {final }}}{m_{\text {initial }}} \cdot 100 \%
$$

Eq. 1

where $m_{\text {inital }}$ is the initial dry weight of the sample and $m_{\text {final }}$ is the final weight of the sample after 3 hrs in water and subsequent drying.

\subsection{Evaluation of intermolecular interactions of chitosan of varying DDA with mucin from bovine submaxillary glands by QCM-D}

Mucin from bovine submaxillary glands was suspended at a concentration of $0.5 \mathrm{mg} / \mathrm{mL}$ in $10 \mathrm{mM}$ acetate buffer $\mathrm{pH} 4.0$ at RT, and stored overnight at $4{ }^{\circ} \mathrm{C}$. Chitosans $(0.2 \mathrm{mg} / \mathrm{mL})$ of various DDA were dispersed in $0.5 \%(\mathrm{v} / \mathrm{v})$ acetic $\mathrm{pH} 5.0$ overnight at RT by gentle stirring. The QCM-D measurements were performed on a E4 Q-sense (Bioline Scientific, Gothenburg, Sweden) according to Wan et al. (2018) with a few modifications. In short, the quartz crystals were immobilized with mucin from bovine submaxillary glands by chemical conjugation by the amine-coupling method 
(Mazzarino, Coche-Guérente, Lemos-Senna, \& Borsali, 2014). The flow rate was $50 \mu \mathrm{L} / \mathrm{min}$ at all times and the temperature was kept at $37{ }^{\circ} \mathrm{C}$. Firstly, the gold-coated quartz crystals were functionalized with carboxylic acid (SAM-COOH) by incubation with $1 \mathrm{mM}$ MUA in ethanol for 12 hrs at RT followed by rinsing in ethanol and drying. In the QCM-D chambers, the conjugation of mucin from bovine submaxillary glands to the sensors was achieved by exposing the sensors to 200 $\mathrm{mM}$ EDC and $50 \mathrm{mM}$ NHS followed by rinsing in ultrapure water. After rinsing the sensors with 10 $\mathrm{mM}$ acetate buffer $\mathrm{pH} 4.0$, they were exposed to $0.5 \mathrm{mg} / \mathrm{mL}$ mucin from bovine submaxillary glands in $10 \mathrm{mM}$ acetate buffer $\mathrm{pH} 4.0$ for $30 \mathrm{~min}$. The sensors were again rinsed with $10 \mathrm{mM}$ acetate buffer $\mathrm{pH} 4.0$ followed by deactivation of unreacted $\mathrm{NH}$-ester by $1 \mathrm{M}$ ETA-HCl in $10 \mathrm{mM}$ Tris buffer $\mathrm{pH}$ 8.5 for $30 \mathrm{~min}$. Finally, prior to the interaction study, the mucin-covered sensors were rinsed firstly in $10 \mathrm{mM}$ acetate buffer $\mathrm{pH} 4.0$ for $15 \mathrm{~min}$ followed by rinsing in $87.9 \mathrm{mM}$ acetate buffer $\mathrm{pH} 5.0$ for $15 \mathrm{~min}$ and then exposed to $0.2 \mathrm{mg} / \mathrm{mL}$ of chitosans in $87.9 \mathrm{mM}$ acetate buffer $\mathrm{pH} 5.0$ of various DDAs for $15 \mathrm{~min}$. The sensors were subsequently rinsed in $87.9 \mathrm{mM}$ acetate buffer $\mathrm{pH} 5.0$ for at least $15 \min$.

\subsection{Compatibility of electrospun chitosan/PEO nanofibers to human buccal TR146 cell monolayers}

The viability of TR146 cells after exposure to nanofibers was evaluated by the MTS/PMS colorimetric assay as previously described (Stie et al., 2019). Briefly, TR146 cells were cultured in Corning Costar ${ }^{\circledR}$ polystyrene culture flasks $\left(175 \mathrm{~cm}^{2}\right.$, Sigma Aldrich, St. Louis, MO, USA) in DMEM supplemented with FBS $(10 \%(\mathrm{v} / \mathrm{v}))$, L-glutamine $(2 \mathrm{mM})$, penicillin $(100 \mathrm{U} / \mathrm{mL})$ and streptomycin $(100 \mu \mathrm{g} / \mathrm{mL})$ in a humidified environment at $37{ }^{\circ} \mathrm{C}$ with $5 \% \mathrm{CO}_{2}$. A total of 100,000 TR146 cells/well were seeded in flat-bottom, transparent 12-well Nunclon ${ }^{\mathrm{TM}}$ delta cell culture-treated plates (Thermo Scientific, Roskilde, Denmark) and cultured for $72 \mathrm{hrs}$ at the aforementioned conditions attaining a confluency of 70-90\%. The cells were washed twice in $2 \mathrm{~mL} 37{ }^{\circ} \mathrm{C}$ hHBSS 
(HBSS with $10 \mathrm{mM}$ HEPES adjusted to $\mathrm{pH} 6.8$ ). The cells were exposed to nanofiber discs of $1.5 \mathrm{mg}$ $( \pm 10 \%)$ submerged in $1.5 \mathrm{~mL}$ hHBSS and incubated for $3 \mathrm{hrs}$ at $37^{\circ} \mathrm{C}$. After exposure, the nanofibers were removed and the cells were washed twice with $2 \mathrm{~mL}$ warm $\left(37^{\circ} \mathrm{C}\right) \mathrm{hHBSS}$. The cells were then incubated at $37{ }^{\circ} \mathrm{C}$ for up to 120 min with $1 \mathrm{~mL}$ reagent solution containing $240 \mu \mathrm{g} / \mathrm{mL}$ MTS and 2.4 $\mathrm{mg} / \mathrm{mL}$ PMS in hHBSS buffer. Subsequently, $100 \mu \mathrm{L}$ samples in quadruplicate of the metabolized solution were transferred from each well to a transparent 96-well plate and the absorbance at $492 \mathrm{~nm}$ was measured in a plate reader (POLARstar OPTIMA, BMG LABTECH, Ortenberg, Germany). The absorbance of the unreacted MTS/PMS solution was defined as the blank (Abs blank), while the control was defined as cells incubated with hHBSS ( $\mathrm{Abs}_{\text {control, }}$ 100\% cell viability). The relative cell viability was determined (Eq. 2):

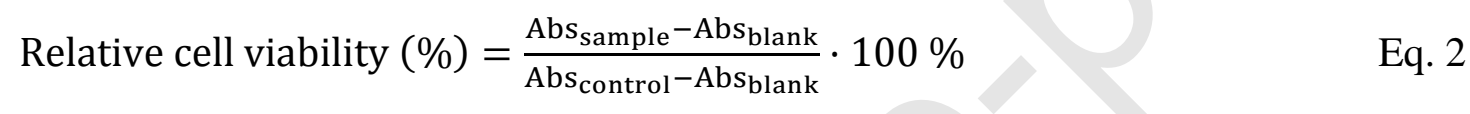

\subsection{Assessment of the adhesion of chitosan/PEO nanofibers to ex vivo porcine sublingual mucosa}

Tongues and ears originating from healthy experimental pigs (approximately 30-60 kg, Danish Landrace/Yorkshire/Duroc) was collected immediately after euthanization and kept in PBS on ice until use on the same day as harvesting of the tissue. The tongues were trimmed to remove underlying tissue and the skin from the back of the ears was gently shaved. The ventral side of the tongues and the skin from the back of the ears were cut to a thickness of 0.25-0.50 mm with an Electric Dermatome (Zimmer Biomet, Albertslund, Denmark) and immediately mounted on microscopy glass slides using Loctite Power Flex gel (Henkel, Ballerup, Denmark) and kept submerged in PBS on ice until measurements. Adhesion testing was conducted on a TA.XT plus texture analyzer (Stable Micro Systems, Godalming, UK) employed with a $50 \mathrm{~N}$ load cell at RT. Discs of a size of $10 \mathrm{~mm}$ consisting of either nanofibers or hydrophobic film were cut with a biopsy puncher and mounted on the probe using double-sided tape. For tests on 'dry tissue', excess buffer on the tissue was removed gently with 
filter paper prior to the analysis. The samples were placed in contact with the tissue for $10 \mathrm{~s}$ by applying a force of $500 \mathrm{~g}$, and then withdrawn with a speed of $10 \mathrm{~mm} / \mathrm{s}$. The work of adhesion was determined as the area under the recorded force versus distance curve using the Exponent software (Stable Micro Systems, Godalming, UK).

\subsection{Swelling of chitosan/PEO nanofibers}

The swelling was determined for a disc of $10 \mathrm{~mm}$ in diameter of either nanofibers or the hydrophobic film retained in metal baskets submerged in $3 \mathrm{~mL}$ ultrapure water for $15 \mathrm{~min}$ at $\mathrm{RT}$. The baskets were drawn from the water and gently blotted on filter paper to remove excess water and the adsorption of water was determined immediately by Eq. 3:

$$
\text { Swelling }(\%)=\frac{\mathrm{m}_{\text {final }}-\mathrm{m}_{\text {intial }}}{\mathrm{m}_{\text {initial }}} \cdot 100 \% \quad \text { Eq. } 3
$$

where $m_{\text {inital }}$ is the initial dry weight of the sample and $m_{\text {final }}$ is the final weight of the sample after 15 min in water.

\subsection{Wettability of tissue by contact angle measurements}

Thin sections of porcine sublingual mucosa or skin were cut and mounted on glass slides as described above. Excess PBS on the surface of the tissue was gently removed using filter paper. Contact angle measurements were performed at RT on a Krüss G2 angle meter (Krüss, Hamburg, Germany) by applying a standing drop of PBS from a $13 \mathrm{G}$ needle on the tissue and measuring the contact angle for the sessile drop with manual baseline fitting using the Drop Shape Analysis software (version 1.90.0.22, Krüss). 


\section{Results and discussion}

\subsection{The DDA of chitosan does not affect the morphology of electrospun chitosan/PEO nanofibers in their dry state}

The effect that the DDA of chitosan on the morphology of chitosan/PEO nanofibers prepared by electrospinning was evaluated by comparing chitosan/PEO nanofibers with chitosan of DDA of 53\%, $71 \%, 82 \%$ or $96 \%$, respectively. The chitosan to PEO ratio (w/w) of the nanofibers were $1: 1$. In all cases, smooth and uniform nanofibers were obtained after electrospinning (Figure 1A). The DDA of chitosan did not affect the size of chitosan/PEO nanofibers electrospun under the selected conditions (Figure 1B). In contrast, Mendes et al., (2018) reported that a small decrease in chitosan DDA from $12 \%$ to $6 \%$ induced a doubling of the diameter of electrospun chitosan/phospholipid fibers. The authors attributed the effect of chitosan DDA on fiber morphology to an increased flexibility of chitosan with increasing DDA (Mendes et al., 2018). The flexibility and charged state of the polymer, which is dependent on the number of repulsive positive charges, can affect its degree of entanglement; a very important property for the electrospinning process.(Shenoy, Bates, Frisch, \& Wnek, 2005) The nanofibers presented by Mendes et al., (2018) consisted of chitosan and phospholipid (asolectin), and the solvent for electrospining was TFA:dichloromethane (30:70) (Mendes et al., 2018). In contrast, to avoid such strong acids and toxic organic compounds, the present chitosan-based nanofibers (Figure 1) were electrospun with PEO as a co-spinning polymer in an aqueous dispersion with dilute aqueous acetic acid $(0.7 \% \mathrm{w} / \mathrm{w})$ as a solvent. Thus, it is evident that solution properties (e.g. viscosity and conductivity) in addition to process and ambient conditions have strong impact on the electrospinning process and hence on the resulting nanofiber morphology (Chronakis, 2010). 


\section{A}

Chitosan-53/PEO

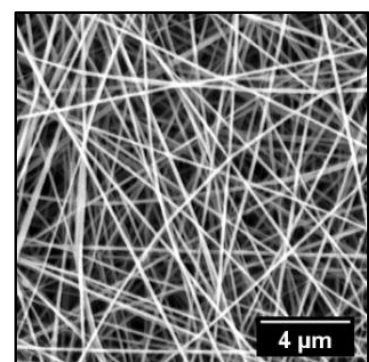

B

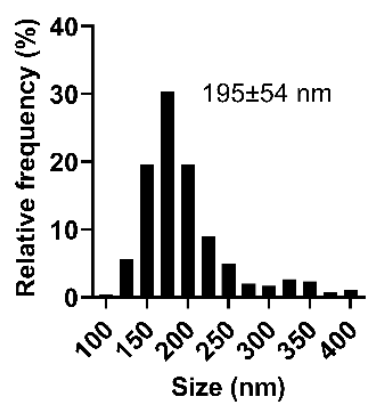

Chitosan-71/PEO
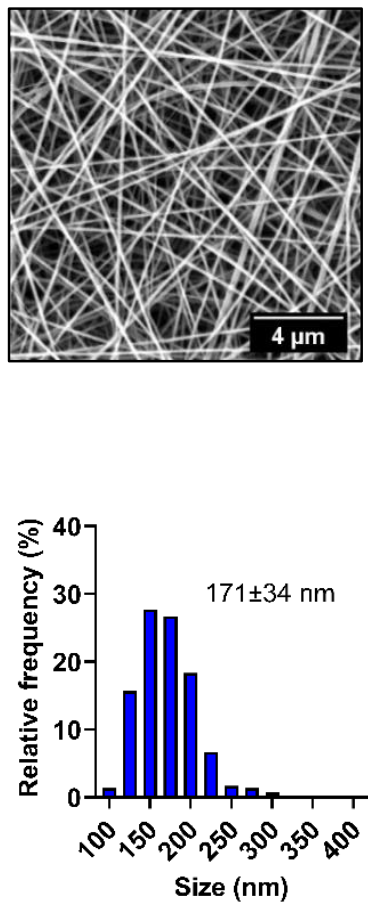

Chitosan-82/PEO
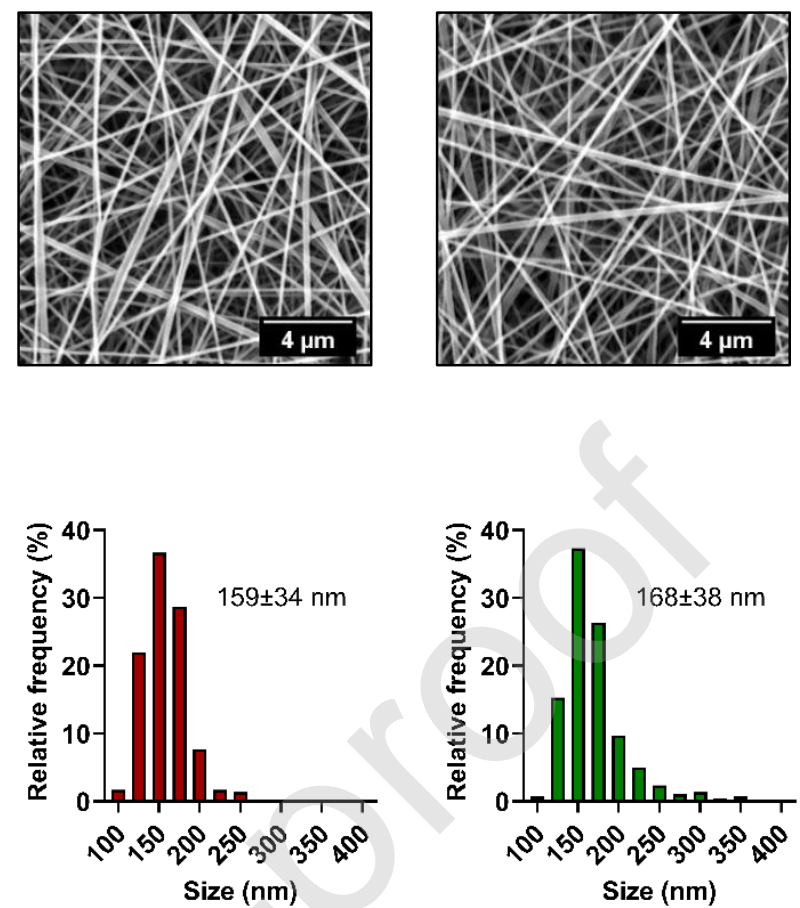

Figure 1. Morphology of chitosan/PEO nanofibers with chitosan of various DDA. A) Representative SEM images of electrospun chitosan/PEO nanofibers with chitosan of various DDA (53\%, 71\%, 82\% or 96\%). 10,000x magnification. B) Size distribution of electrospun chitosan/PEO nanofibers with chitosan of various DDA. Mean diameters \pm standard deviations (SD) are given. $\mathrm{N}=3, \mathrm{n}=100$, where $\mathrm{N}$ represents the number of individual fiber mats and $\mathrm{n}$ is the numbers of individual fibers measured per fiber mat.

FTIR analysis with ATR confirmed the presence of chitosan and PEO in the nanofibers prepared by electrospinning of chitosan with various DDA and PEO in accordance with Stie et al., (2019). Chitosan is amongst others characterized by C-N stretching at $1000 \mathrm{~cm}^{-1}$ (Figure 2A). A clear doublet at $1650 \mathrm{~cm}^{-1}$ (amide I) and $1550 \mathrm{~cm}^{-1}$ (amide II) is present in the spectra of chitosan-53 and for nanofibers consisting hereof (chitosan-53/PEO, Figure 2B). The absorption from the amide bond in these regions of the spectrum weakens as the DDA of chitosan increases. In addition, a single peak at $1590 \mathrm{~cm}^{-1}$ appears in the FTIR spectrum as the DDA of chitosan increases (chitosan-96, Figure 2B), which is characteristic for the N-H bending of the amine (Feng, Liu, Zhao, \& Hu, 2012). PEO is clearly identified by the C-O-C stretching at $1100 \mathrm{~cm}^{-1}$ in the FTIR absorbance spectrum. 
A

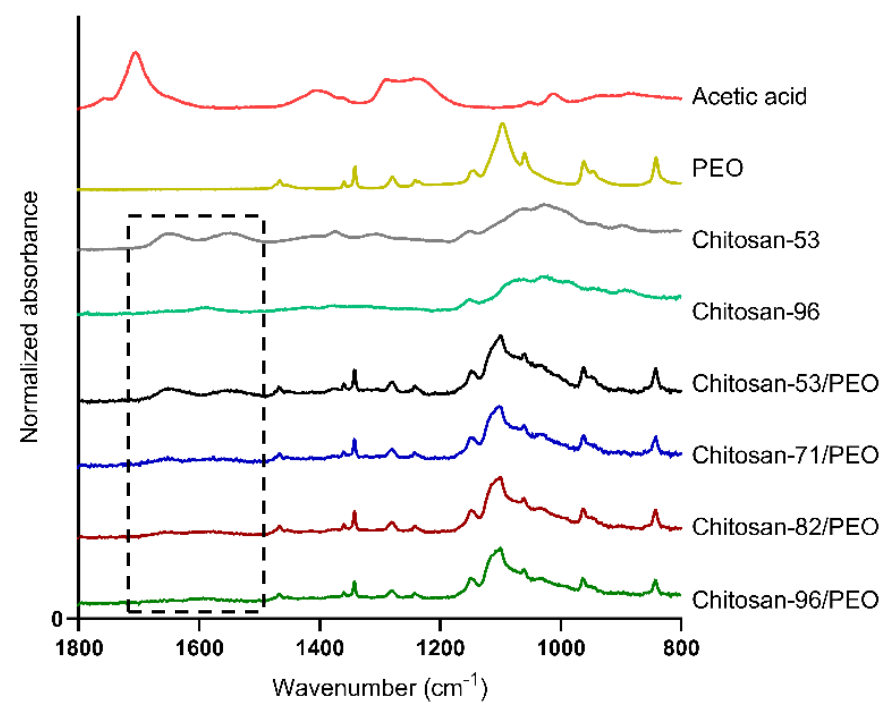

B

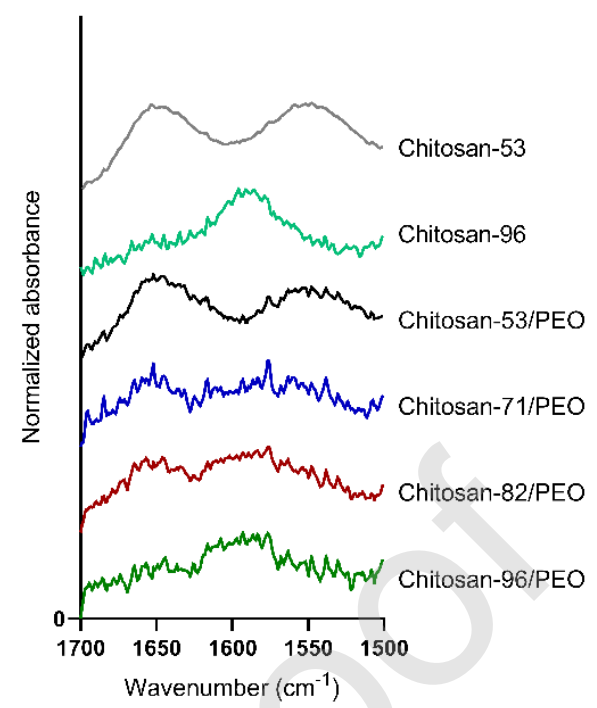

Figure 2. Composition analysis by FTIR with ATR of electrospun chitosan/PEO nanofibers prepared with chitosan of various DDA. A) Representative FTIR spectra of acetic acid, PEO, chitosan-53, chitosan-96 and electrospun chitosan/PEO nanofibers consisting of chitosan with various DDA $(53 \%, 71 \%, 82 \%$ or $96 \%)$. B) Representative FTIR with ATR spectra in the region of 1700 $1500 \mathrm{~cm}^{-1}$ of chitosan-53, chitosan-96 and chitosan/PEO nanofibers electrospun with chitosan of various DDA. N=2, n=2, where N represents the number of individual samples, and $\mathrm{n}$ is the numbers of measurements per sample.

\subsection{Morphological stability of wetted chitosan/PEO nanofibers is dependent on the DDA of}

\section{chitosan}

PEO is a water-dispersible polymer, and neat PEO nanofibers disintegrate completely within minutes when exposed to water (Stie et al., 2019). Nanofibers consisting of chitosan with a DDA of 53\%, $71 \%, 82 \%$ or $96 \%$ all displayed an average weight loss of $49-53 \%$, which corresponds to the content of PEO in the nanofibers (Figure 3A); chitosan-53/PEO showed lowest reproducibility (i.e. larger SD) in weight loss after exposure to water compared to the other chitosan nanofibers tested. Supporting this, we have previously demonstrated that after exposure to water, PEO is completely removed from chitosan/PEO nanofibers made with $30 \%$ (w/w) chitosan-96 resulting in neat chitosan nanofibers (Stie et al., 2019). Interestingly, some of the chitosan/PEO nanofibers lost their fibrous structure completely (chitosan-53/PEO and chitosan-71/PEO) or partially (chitosan-82/PEO), when exposed to water for $3 \mathrm{hrs}$. In contrast, chitosan-96/PEO nanofibers containing chitosan with the highest DDA used in this study retained their structure after a 3 hrs exposure to water (Figure 3B). 
To explain the loss of fibrous structure with decreasing DDA of chitosan after exposure of the nanofibers to water, it is important to realize that chitosan with a high DDA is dispersible only in slightly acidic medium $(\mathrm{pH}<6)$ and not at neutral $\mathrm{pH}$, whereas chitosan with a DDA of around 50\% is dispersible at neutral pH (Sogias, Khutoryanskiy, \& Williams, 2010). The solid state of chitosan is closely related to the ability of the polymer to be dispersed in water (Sogias et al., 2010). Chitosan with a high DDA has a high degree of crystallinity and is therefore less soluble at neutral $\mathrm{pH}$ in comparison to half-acetylated chitosan, which, by use of X-ray diffraction, was shown to be the least crystalline compared to chitosans of higher DDAs, and half-acetylated chitosan therefore had the best dispersability at neutral pH (Sogias et al., 2010).

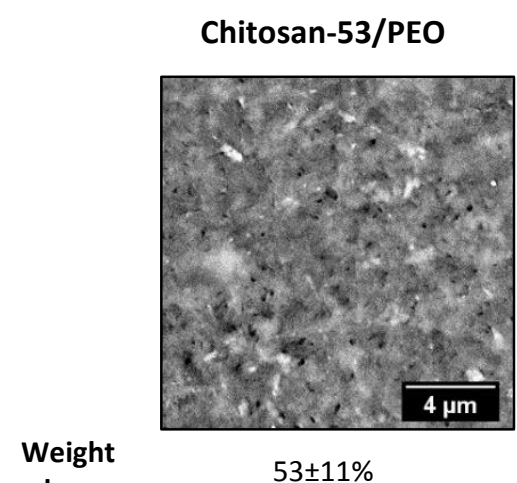

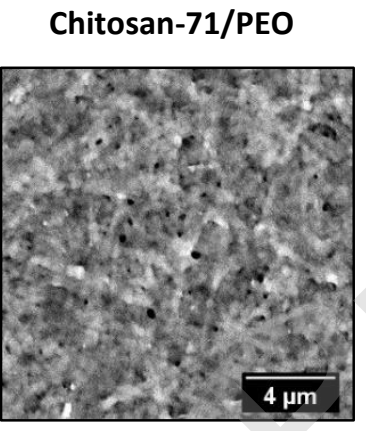

$50 \pm 1 \%$

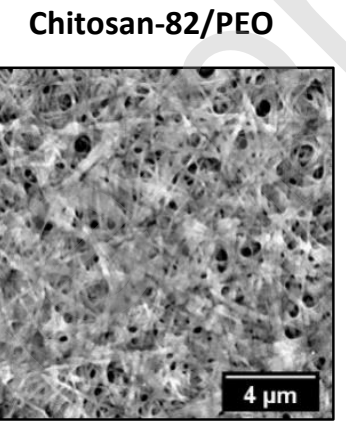

$50 \pm 2 \%$
Chitosan-96/PEO

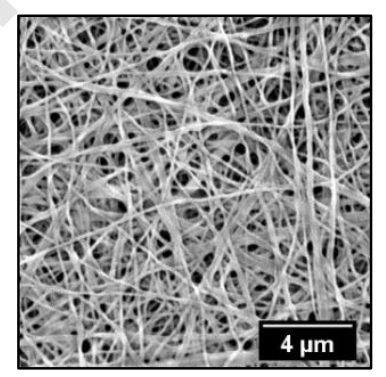

$49 \pm 3 \%$

Figure 3. Aqueous stability of chitosan/PEO nanofibers electrospun with chitosan of various DDA. Representative SEM images (dry) and weight loss of chitosan/PEO nanofibers electrospun with chitosan of various DDA (53\%, 71\%, 82\% or 96\%) after exposure to water for 3 hrs. 10,000x magnification. Weight loss is given as mean $\pm \mathrm{SD}, \mathrm{N}=3$, where $\mathrm{N}$ is the number of individually prepared nanofiber mats tested.

For a number of proposed medical applications of electrospun nanofibers, tuning of the morphological stability upon wetting with aqueous medium is crucial. For example, electrospun nanofibers have gained much interest in the field of regenerative medicine due their three-dimensional structure and porosity allowing for exchange of nutrients and waste. The fibrous network resembles the extracellular matrix and has therefore been proposed as scaffolds to facilitate cell penetration, proliferation and differentiation (Qasim et al., 2018). In addition, chitosan-based nanofibers have been proposed as wound dressings as the airy, semi-permeable and porous network of nanofibers 
allow exchange of gas and water between the wound and the surrounding tissue. As an example, chitosan/PEO nanofibers with chitosan of DDA of $85 \%$ were shown to stimulate skin regeneration in patients suffering from IIIa and IIIb degree burns (Kossovich, Salkovskiy, \& Kirillova, 2010). Nanofibers electrospun from chitosan with a high DDA may therefore be superior to chitosan with low DDA to maintain their fibrous network when exposed to a moist environment. For sustained and controlled release of an encapsulate drug, drug delivery systems with improved water stability is preferred to allow a longer duration of drug release, as for example demonstrated by Laha et al., (2017), who achieved zero-order drug release from water-stable multilayered gelatin nanofibers during 48 hrs (Laha et al., 2017). Films and tablets that disintegrate fast when exposed to water are beneficial for treatments that require a fast onset of action and can be taken with very little or no water as for example fastmelt tablets with analgesics or antiphychotics (e.g. Zyprexa®). Differences in the morphological stability of the nanofibers in aqueous medium may have significant effects on the drug release kinetics. This property could provide a possibility for tuning chitosan/PEO nanofibers to an optimal release profile by varying the DDA of chitosan, and thereby changing the properties of the nanofibers.

\subsection{Chitosan/PEO nanofibers are biocompatible to in vitro TR146 cell monolayers}

The use of biocompatible excipients is significant for future clinical translation of chitosan/PEO nanofibers. It is worth noting that the chitosan/PEO nanofibers were produced from biocompatible and biodegradable polymers at mild acidic conditions using dilute aqueous acetic acid as a solvent. To confirm the biocompatibility of chitosan/PEO nanofibers regardless of the DDA of chitosan, monolayers of the human non-keratinized epithelial TR146 cells of buccal origin were exposed for 3 hrs to nanofibers prepared with various DDA $(53 \%, 71 \%, 82 \%$ or $96 \%$, respectively). The effect on the relative cell viability was compared to that of cells exposed to the buffer as a control. No effect 
on cell viability was observed for any of the investigated electrospun chitosan/PEO nanofibers relative to the control (Figure 4).

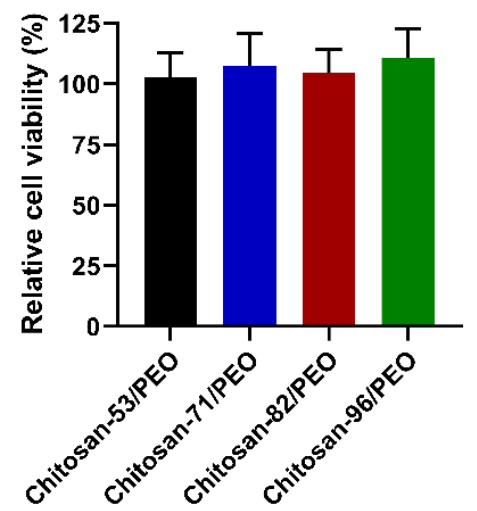

Figure 4. Evaluation of the in vitro compatibility of chitosan/PEO nanofibers with chitosan of various DDA to TR146 cell monolayers relative to control (isotonic buffer, $\mathbf{p H}$ 6.8). Relative viability is given as mean $\pm \mathrm{SD}, \mathrm{N}=3, \mathrm{n}=3$, where $\mathrm{N}$ represents the number of cell passages, and $\mathrm{n}$ is the number of samples tested per passage.

\subsection{Increasing DDA of chitosan induces stronger binding to mucin from bovine submaxillary} glands

Intermolecular interactions can be monitored in real time by the QCM-D technique. In short, changes in frequency $(\Delta \mathrm{F})$ is proportional to adsorbed mass on the QCM-D sensor, whereas changes in dissipation $(\Delta \mathrm{D})$ and spreading of the overtones are associated with a change in the mechanical properties of the material on the sensor. To evaluate the effect of the DDA of chitosan on its intermolecular interaction with mucin, bovine submaxillary mucin was chemically immobilized on gold quartz sensors by NHS-functionalization (Mazzarino et al., 2014; Wan et al., 2018), and the adsorption of chitosans of DDA between 53\% and 96\% was evaluated (Figure 5). A significant decrease in $\Delta \mathrm{F}$ and an increase $\Delta \mathrm{D}$ were observed after exposure of the functionalized sensor to mucin, which suggests that a viscoelastic layer of mucin was bound to the surface of the sensor (Figure 5A). Changes in frequency are not only related to the adsorption of mucin to the QCM-D sensor, but also to the mass of associated water. Interestingly, chemically immobilized mucin formed a rigid layer at $\mathrm{pH} 4$ after deactivation, but the mucin layer was in general more viscoelastic (increase 
in $\Delta \mathrm{D}$ ) and more hydrated (decrease in $\Delta \mathrm{F}$ ) at higher $\mathrm{pH}$ values. This indicates that mucin from bovine submaxillary glands may undergo conformational changes dependent on the $\mathrm{pH}$. Chitosan, especially with a high DDA, does not form a clear dispersion at neutral $\mathrm{pH}$. The QCM-D experiments were therefore conducted at mild acidic conditions ( $\mathrm{pH}$ 5) to avoid precipitation of chitosan during the analysis.

A significant decrease in $\Delta \mathrm{F}$ and an increase $\Delta \mathrm{D}$ were observed after exposure of the established mucin layer to each of the chitosans, which is evident of an interaction between mucin and the chitosans (Figure 5B). No significant changes in neither $\Delta \mathrm{F}$ nor $\Delta \mathrm{D}$ were observed after rinsing the mucin-chitosan layers with buffer (Figure 5B). This suggests that chitosan, independent of the DDA, binds strongly and irreversibly to the mucin layer under the presented conditions. The slope of a $\Delta \mathrm{D} / \Delta \mathrm{F}$ plot (Figure 5C) provides information on the stiffness of the established mucin-chitosan layers, which is an indicator for the strength of the interaction between the glycoproteins and the polymers (Feiler, Sahlholm, Sandberg, \& Caldwell, 2007). The most stiff layer and thus the strongest interaction with mucin was found for chitosan-96 in comparison to chitosans with a lower DDA as shown by less steep slopes in the $\Delta \mathrm{D} / \Delta \mathrm{F}$ plot (Figure 5C-D).

In general, mucin has a net negative charge at $\mathrm{pH}$ values above $\mathrm{pH} 2$ and chitosan has a net positive charge at acidic $\mathrm{pH}$. The experiments were conducted at $\mathrm{pH}$, which thus facilitates electrostatic interactions between the cationic chitosan and the anionic mucin. It was therefore not surprising that chitosan-96 with the highest DDA and thus with the highest number of positive charges of the tested chitosans interacted most strongly with the mucin layer. However, chitosan-53 despite its lower DDA also interacts strongly with mucin from bovine submaxillary glands. The interaction of chitosan with mucin may also be driven by hydrophobic interactions and hydrogen bonding as has been suggested for the interaction of chitosan with gastric mucin (Sogias, Williams, \& Khutoryanskiy, 2008). 
Although chitosan is well-known for being mucoadhesive, a greater understanding of the underlying mechanisms of the interaction of chitosan and especially of chitosan-based drug delivery systems with mucin is still missing. A significant number of the reported studies on the mucoadhesive properties of chitosan focus on the interaction of chitosan with highly purified mucin derived from porcine gastric mucus. It is important to note that the expressed mucin glycosylation pattern varies depending on the site of origin i.e., the gastric mucosa is rich in MUC5AC and MUC6 (Nordman et al., 2002), whereas MUC5b and MUC7 are the most abundant mucins present in saliva (Hannig, Hannig, Kensche, \& Carpenter, 2017). Furthermore, the methods used for mucin extraction and purification may induce variabilities in the degree of mucin purity. Also, inter-species variations may be considered. For studies on oromucosal drug delivery, it is therefore relevant to use a source of mucin derived from the oral cavity as differences in molecular structure, glycosylation pattern and purity of the mucin may influence the interactions. 
A

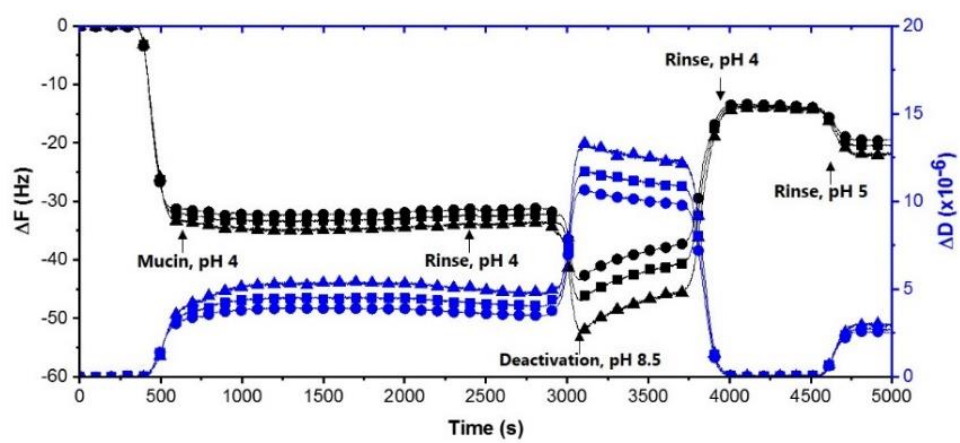

B
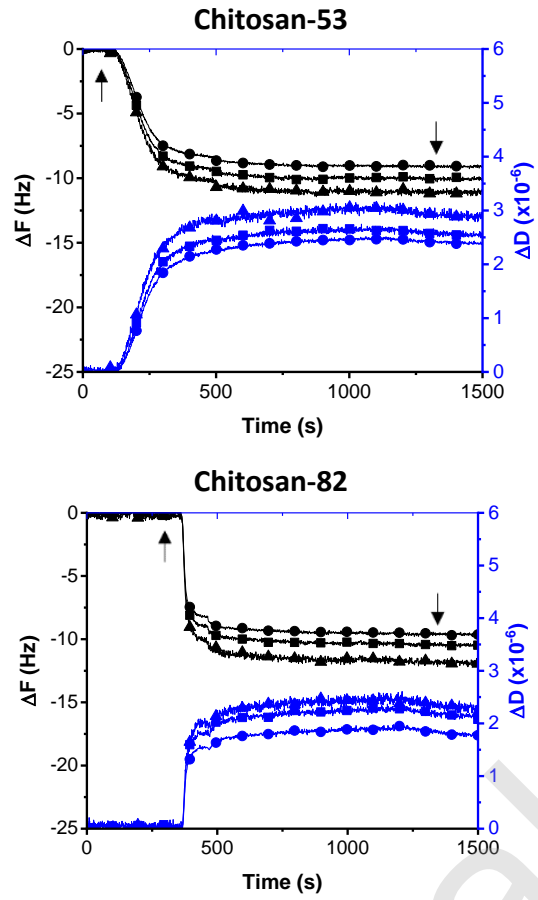

C

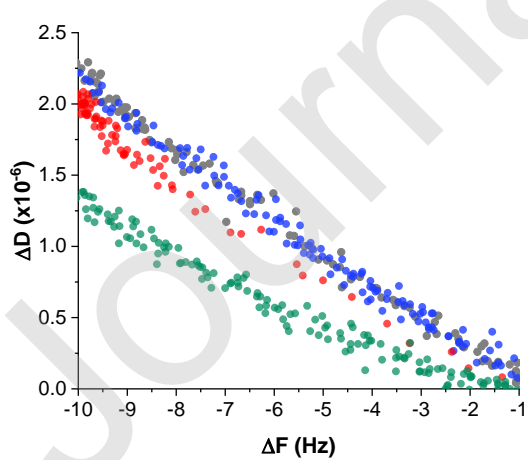

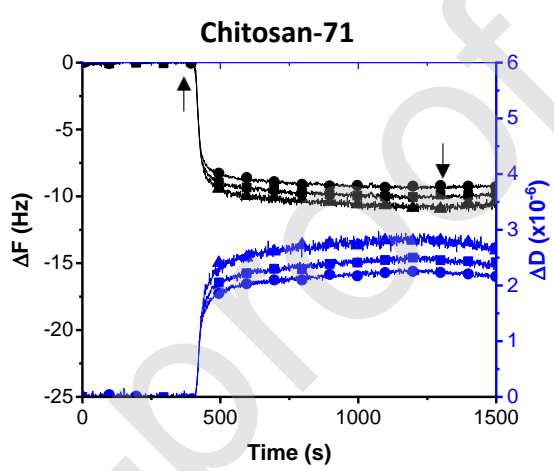

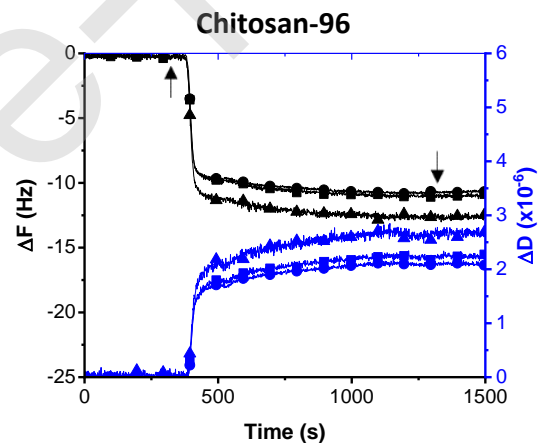

D

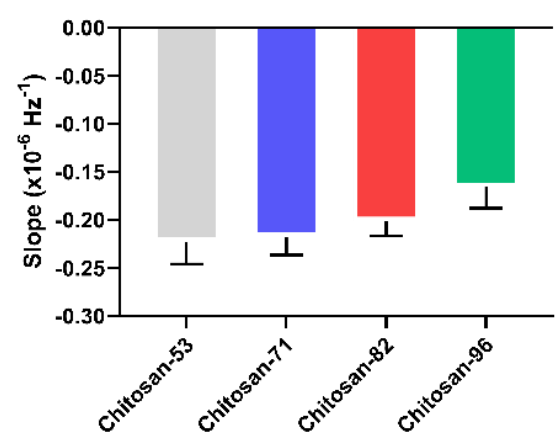

Figure 5. Interaction of mucin from bovine submaxillary glands with chitosan of increasing DDA evaluated by the QCM-D technique. A) Representative QCM-D measurement of changes in frequency $(\Delta \mathrm{F})$ and dissipation $(\Delta \mathrm{D})$ during the adsorption of mucin from bovine submaxillary glands onto the QCM-D sensor achieved by NHS functionalization. The fifth (circles), seventh (squares) and ninth (triangle) overtone are shown. B) Representative QCM-D measurements after exposing the established mucin layer to chitosans of various DDA (53-96\%). Arrows indicate the introduction of chitosan and subsequent rinsing. C) Representative $\Delta \mathrm{D} / \Delta \mathrm{F}$ plot (fifth overtone) of the adsorption of chitosans (DDA of 53-96\%) to the mucin layer. The interpretation of color is given in D), which shows the slope of the linear region of the $\Delta F / \Delta D$ plot represented in Figure $5 C$. Results are presented as mean $\pm S D$. $N=2, n=2$, where $\mathrm{N}$ represent the number of individual samples and $\mathrm{n}$ is the number of repeats per sample. 


\subsection{Chitosan/PEO nanofibers adhere strongly to ex vivo porcine sublingual mucosa}

The mucoadhesive properties of chitosan/PEO nanofibers were evaluated by measuring the work of adhesion of nanofibers containing chitosan with various DDA to ex vivo sublingual mucosa. The sublingual region of rodents is keratinized and are therefore not an optimal model for the sublingual non-keratinized mucosa in humans (Thirion-Delalande et al., 2017). Larger animals such as pigs have a non-keratinized sublingual mucosa with larger rete pegs comparable to that of humans and thus, the tissue constitute an appropriate model for the human sublingual tissue (Thirion-Delalande et al., 2017). Tissue sections of ex vivo porcine sublingual mucosa were obtained by the use of an electric dermatome (Figure 6A), and they were mounted on a glass support, and hydrated in isotonic buffer prior to analysis (Figure 6B). Chitosan/PEO nanofibers showed strong adhesion to the sublingual tissue compared to the hydrophobic ethyl cellulose film (non-mucoadhesive control) (Figure 6C). No differences were found in the adhesiveness of the different electrospun chitosan/PEO nanofibers irrespective of the DDA of the chitosan used.

A

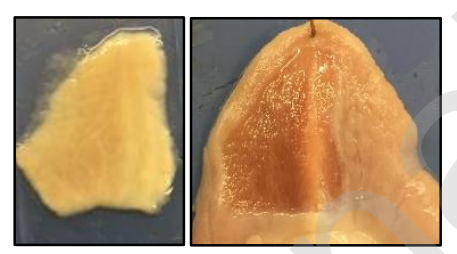

B

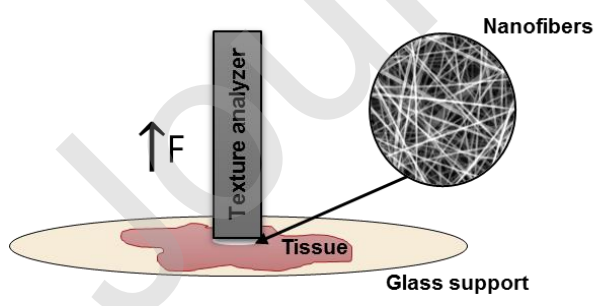

C

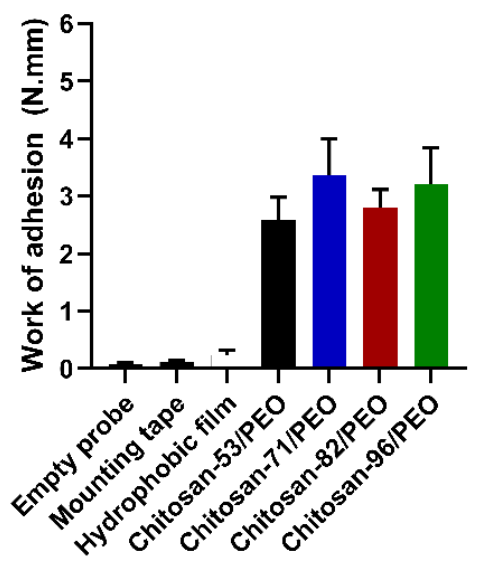

Figure 6. Adhesion of electrospun chitosan/PEO nanofibers to ex vivo porcine sublingual mucosa. A) Tissue sections of ex vivo porcine sublingual mucosa were obtained using an electric dermatome. The images display a thin section of ex vivo porcine sublingual mucosa (left) and the ventral side of a porcine tongue after removal of the epithelium (right). B) Experimental setup. The work of adhesion of chitosan/PEO nanofibers from ex vivo porcine sublingual mucosa mounted on a glass support was recorded by a texture analyzer. C) Work of adhesion of electrospun chitosan/PEO nanofibers with chitosan of various DDA (53\%, 71\%, 82\% or 96\%). The empty probe, mounting tape and a hydrophobic film were included as controls. Results are presented as mean $\pm \mathrm{SD}$. N=4-5, $\mathrm{n}=1-4$, where $\mathrm{N}$ represents the number of biological repeats, and $\mathrm{n}$ is the number of measurements per individual tissue. 
To the best of our knowledge, this is the first report of a quantitative evaluation of the work of adhesion of chitosan/PEO nanofibers to fresh sublingual mucosa. In general, only few studies have investigated the mucoadhesive properties of electrospun nanofibers for oromucosal application. For example, Samprasit et al., (2015) evaluated the mucoadhesion of chitosan/PVA nanofibers to ex vivo porcine buccal mucosa and found that nanofibers with thiolated chitosan were significantly more mucoadhesive, as they required two times the force of detachement from the tissue. Mašek et al., (2017) reported that an adhesive film with chitosan/PEO nanofibers as a reservoir of vaccine nanoparticles adhered to porcine sublingual mucosa for at least two hours in vivo. Close adhesion of the drug delivery system to the site of application is expected to lead to improved drug absorption as a result of longer residence time, and hereby an overall increase in drug bioavailability. Furthermore, close adhesion of the drug delivery system to the tongue or other affected areas of the oral mucosa could enable better local treatment of lesions and ulcerations in the oral cavity. Also, coverage of the lesion with a mucoadhesive electrospun patch may protect the sensitive area and enhance wound healing.

\subsection{The mucoadhesive properties of chitosan/PEO nanofibers is related to their swelling capability}

The work of adhesion of chitosan-96/PEO nanofibers from ex vivo porcine sublingual mucosa was compared to the work of adhesion of nanofibers to a non-mucosal tissue, i.e. ex vivo porcine skin. As the work of adhesion of electrospun chitosan/PEO nanofibers to ex vivo porcine sublingual mucosa was not dependent on the DDA of chitosan, only nanofibers with the highest DDA of chitosan, chitosan-96/PEO, were included in the following experiment. Thus, the work of adhesion of chitosan96/PEO nanofibers was tested on tissue wetted in isotonic buffer as in Figure 6 or on dry tissue (tissue gently wiped to remove excess buffer) (Figure 7A). As expected, chitosan-96/PEO nanofibers adhered significantly stronger to ex vivo porcine sublingual mucosa compared to ex vivo porcine skin, 
even when the skin was wetted. Interestingly, the work of adhesion of chitosan-96/PEO nanofibers to the sublingual mucosa was not significantly $(\mathrm{p}>0.25)$ affected by wetting of the tissue. In contrast, the adhesiveness of the nanofibers to wetted skin was significantly stronger than for skin samples where surplus of liquid was removed from the surface. The electrospun chitosan/PEO nanofiber appeared hydrated and swollen after detachment from the tissue during adhesive testing. Furthermore, by visually inspection, especially the sublingual tissue appeared slightly dehydrated in the area of contact after removal of the nanofibers. This strongly indicates that the mucoadhesive properties of the nanofibers are dependent on their ability to swell and dehydrate the tissue upon contact. Differences in adhesiveness of the nanofibers to sublingual mucosa and skin were therefore explained by differences in the wettability of the mucosal or non-mucosal tissue. The wettability of the surface can be described by the contact angle between the liquid and the surface. To demonstrate differences in the wettability between the sublingual mucosa and the skin, the contact angle of wetted ex vivo porcine sublingual mucosa was compared to that of ex vivo porcine skin. The contact angle of PBS on ex vivo porcine sublingual mucosa was lower than for ex vivo porcine skin, which demonstrates better wettability of the sublingual mucosal tissue compared to that of the skin. The contact angles reported here should be taken as relative values under the presented experimental condition, as the extent of tissue hydration may be different compared to the in vivo situation, and the presence of an intact saliva pellicle may affect the hydrophilicity of the tongue in vivo (Ranc et al., 2006). Methodologically, it is worth noting the importance of precise even mounting of the tissue, as uneven mounting of the tissue on the glass support changes the area of contact between tissue and nanofibers, and artificial roughness of the tissue could induce artifacts in the force of adhesion measurements by the presented method (Figure 7B). 
A

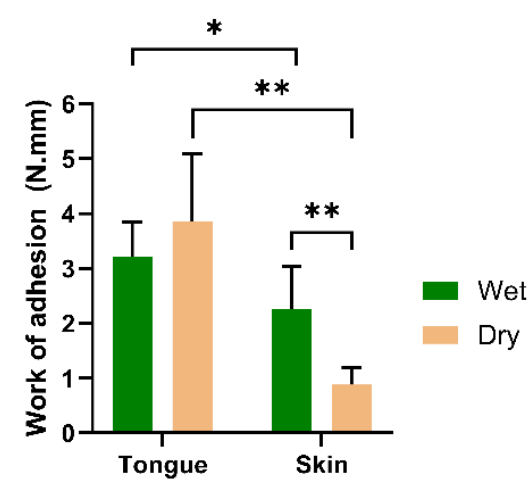

B
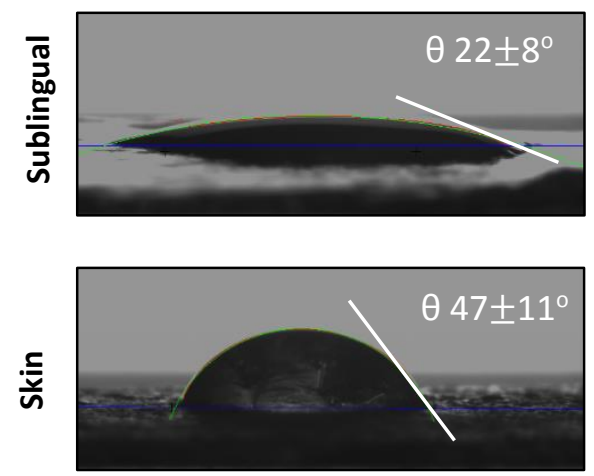

C

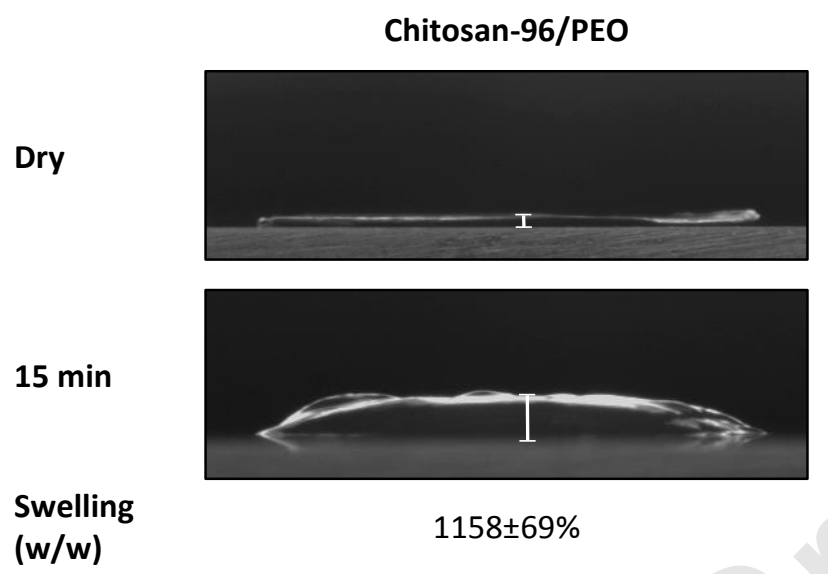

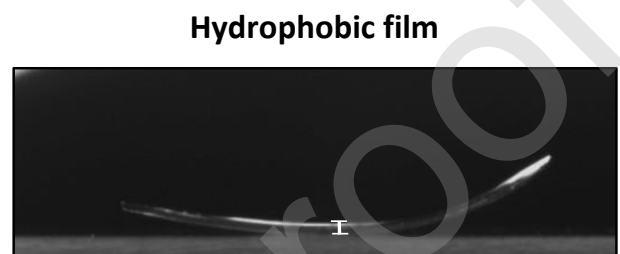

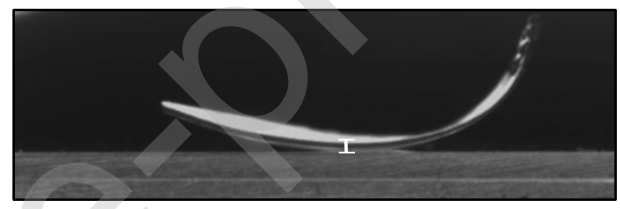

$12 \pm 4 \%$

Figure 7. Wetting and swelling of chitosan/PEO nanofibers facilitate adhesion to porcine sublingual mucosa ex vivo. A) Work of adhesion of electrospun chitosan-96/PEO nanofibers to ex vivo porcine sublingual tissue or skin, both either dried or wetted in PBS. $\mathrm{N}=2, \mathrm{n}=3-5$, where $\mathrm{N}$ represents the number of biological repeats, and $\mathrm{n}$ is the number of measurements per individual tissue. ${ }^{*} \mathrm{p}<0.05$, $* * \mathrm{p}<0.005$. B) Contact angle measurements of PBS on ex vivo porcine sublingual mucosa or skin. Results are presented as mean \pm SD. $\mathrm{N}=3, \mathrm{n}=2-3$, where $\mathrm{N}$ represent the number of biological replicates, and $\mathrm{n}$ is the number of measurements per tissue. C) Representative images (side-view) of the swelling of a disc (diameter $10 \mathrm{~mm}$ ) of either chitosan-96/PEO nanofibers or a hydrophobic film before and after exposure to water for $15 \mathrm{~min}$. White bars mark the thickness of the discs before and after exposure to water. The swelling of the samples are given as mean $\pm \mathrm{SD}$. $\mathrm{N}=3$, where $\mathrm{N}$ represent the number of repeats.

Indeed, chitosan/PEO nanofibers displayed exceptional swelling abilities and specifically swell more than $1000 \%$ (w/w) after exposure to water for only $15 \mathrm{~min}$ as presented in Figure 7C. In contrast, the hydrophobic film based on ethyl cellulose used as a non-mucoadhesive control absorbed only limited amount of water $(12 \pm 4 \%)$ under the same conditions. Although, Chitosan-71/PEO nanofibers showed almost complete loss of fibrous structure in water (Figure 3), these nanofibers also displayed a high degree of swelling in contact with water $(1132 \pm 36 \%)$. Importantly, the extraordinary swelling ability of the nanofibers is attributed to the fibers high surface-area-to-volume ratio in combination with the hygroscopic nature of chitosan. It is worth noting that only minute amounts of fluid, i.e. only that 
originating from the sublingual tissue, was required to induce swelling of the chitosan/PEO nanofibers inducing strong adherence to sublingual mucosa ex vivo. Thus, application as a mucoadhesive material may be promising also for dry mouth conditions.

No theory alone embraces the full mechanism of mucoadhesion, and a consideration of multiple factors is required to evaluate and describe the mucoadhesive properties of a drug delivery system in depth (Peppas, Thomas, \& McGinty, 2009). As mentioned, electrospun nanofibers have a high surface-area-to-volume ratio, which improves swelling, but also provides a good contact area for adhesion to mucosal surfaces. Hygroscopic polymers such as chitosan with gel-forming abilities are capable of partially dehydrating the mucosal surface at the contact area, as described above, which creates strong adhesiveness when the equilibrium in water absorption from the mucosa to the adhesive drug delivery system is reached (Peppas et al., 2009). Thus, the adhesive properties can to a great extent be described by the ability of the chitosan/PEO nanofibers to swell, spread and hereby establish the initial close contact with a wetted sublingual surface. Swelling of the drug delivery system furthermore enables entanglement of the chitosan in the nanofibers with molecules such as mucins, which are found on the mucosal surface. Accordingly, an important role may be represented by the molecular interactions between chitosan and mucin molecules, as shown by QCM-D in Figure 5, potentially further strengthening the adhesion of the drug delivery system to sublingual mucosa. 


\section{Conclusion}

The present study evaluated the mucoadhesive properties of electrospun chitosan/PEO nanofiber patches containing chitosan of various DDA (53-96\%) intended for sublingual application. The DDA of chitosan had no significant effect on the morphology of the electrospun nanofibers prepared in biocompatible aqueous medium, which were all $<250 \mathrm{~nm}$ in width, smooth and without artifacts such as beading. Interestingly, the DDA of chitosan affected the morphological stability of the nanofibers in water, as nanofibers containing chitosan of low DDA completely or partially lost their fibrous structure in water. In contrast, nanofibers consisting of chitosan with the highest DDA tested (96\%) maintained their fiber network after exposure to water for $3 \mathrm{hrs}$. All chitosans were found to interact strongly with mucin from bovine submaxillary glands independently of their DDA, although the strongest intermolecular interaction with mucin was found for chitosan of the highest DDA. Finally, we for the first time demonstrate excellent adhesiveness of chitosan/PEO nanofibers to porcine sublingual mucosa ex vivo by measuring the work of adhesion of the nanofibers to the sublingual mucosal tissue. Importantly, the swelling of the nanofibers and dehydration of the mucosal tissue upon contact were found to be highly significant for the mucoadhesive properties of electrospun chitosan/PEO nanofibers.

Author statement

Mai Bay Stie: Conceptualization, Methodology, Formal analysis, Investigation, Writing - Original Draft, Visualization, Project administration. Johan Ring Gätke: Methodology, Investigation, Writing - Review and Editing. Feng Wan: Methodology, Investigation, Writing - Review and Editing. Ioannis S. Chronakis: Conceptualization, Validation, Writing - Review and Editing, Supervision. Jette Jacobsen: Conceptualization, Validation, Writing - Review and Editing, Supervision. Hanne Mørck Nielsen: Conceptualization, Validation, Writing - Review and Editing, Supervision, Funding acquisition. 


\section{Conflicts of interests}

The authors declare no conflict of interest.

\section{Acknowledgement}

MBS, JRG, HMN, ISC and JJ acknowledge The Danish Council for Independent Research; Technology and Production [DFF-6111-00333](MBS) for funding, and the Novo Nordisk Foundation [Grand Challenge Program; NNF16OC0021948] for supporting this project. FW acknowledges funding from the Danish Council for Independent Research, Technology and Production Sciences [DFF-4093-00062] and the Lundbeck Foundation [R264-2017-3404]. Department of Experimental Medicine at University of Copenhagen is greatly acknowledged for assistance providing porcine tissue for the ex vivo studies. Louise Kjærulff and Dan Stærk from Department of Pharmacy at University of Copenhagen and Mikkel Boas Thygesen from Department of Chemistry at University of Copenhagen are acknowledged for their assistance with NMR analysis. The authors thank LEO Pharma for availability of the dermatome equipment. 


\section{References}

Bernkop-Schnürch, A., \& Dünnhaupt, S. (2012). Chitosan-based drug delivery systems. European Journal of Pharmaceutics and Biopharmaceutics, 81(3), 463-469. https://doi.org/10.1016/j.ejpb.2012.04.007

Chronakis, I. S. (2010). Micro-/Nano-Fibers by Electrospinning Technology. Micro-Manufacturing Engineering and Technology, 264-284. https://doi.org/10.1016/B978-0-8155-1545-6.00016-8

Clitherow, K. H., Murdoch, C., Spain, S. G., Handler, A. M., Colley, H. E., Stie, M. B., ... Jacobsen, J. (2019). Mucoadhesive Electrospun Patch Delivery of Lidocaine to the Oral Mucosa and Investigation of Spatial Distribution in a Tissue Using MALDI-Mass Spectrometry Imaging. Molecular Pharmaceutics, 16(9), 3948-3956. research-article. https://doi.org/10.1021/acs.molpharmaceut.9b00535

Duan, B., Dong, C., Yuan, X., \& Yao, K. (2004). Electrospinning of chitosan solutions in acetic acid with poly(ethylene oxide). Journal of Biomaterials Science, Polymer Edition, 15(6), 797811. https://doi.org/10.1163/156856204774196171

Feiler, A. A., Sahlholm, A., Sandberg, T., \& Caldwell, K. D. (2007). Adsorption and viscoelastic properties of fractionated mucin (BSM) and bovine serum albumin (BSA) studied with quartz crystal microbalance (QCM-D). Journal of Colloid and Interface Science, 315(2), 475-481. https://doi.org/10.1016/j.jcis.2007.07.029

Feng, F., Liu, Y., Zhao, B., \& Hu, K. (2012). Characterization of half N-acetylated chitosan powders and films. Procedia Engineering, 27(2011), 718-732. https://doi.org/10.1016/j.proeng.2011.12.511

Geng, X., Kwon, O.-H., \& Jang, J. (2005). Electrospinning of chitosan dissolved in concentrated acetic acid solution. Biomaterials, 26(27), 5427-5432.

https://doi.org/10.1016/j.biomaterials.2005.01.066

Hannig, C., Hannig, M., Kensche, A., \& Carpenter, G. (2017). The mucosal pellicle - An underestimated factor in oral physiology. Archives of Oral Biology, 80, 144-152. https://doi.org/10.1016/j.archoralbio.2017.04.001

Khutoryanskiy, V. V. (2011). Advances in Mucoadhesion and Mucoadhesive Polymers. Macromolecular Bioscience, 11(6), 748-764. https://doi.org/10.1002/mabi.201000388

Kossovich, L. Y., Salkovskiy, Y., \& Kirillova, I. V. (2010). Electrospun chitosan nanofiber materials as burn dressing. IFMBE Proceedings, 31, 1212-1214. https://doi.org/10.1007/9783-642-14515-5_307

Kraan, H., Vrieling, H., Czerkinsky, C., Jiskoot, W., Kersten, G., \& Amorij, J.-P. (2014). Buccal and sublingual vaccine delivery. Journal of Controlled Release, 190, 580-592. https://doi.org/10.1016/j.jconrel.2014.05.060

Laha, A., Sharma, C. S., \& Majumdar, S. (2017). Sustained drug release from multi-layered sequentially crosslinked electrospun gelatin nanofiber mesh. Materials Science and Engineering: C, 76, 782-786. https://doi.org/10.1016/j.msec.2017.03.110

Lancina, M. G., Shankar, R. K., \& Yang, H. (2017). Chitosan nanofibers for transbuccal insulin delivery. Journal of Biomedical Materials Research Part A, 105(5), 1252-1259. https://doi.org/10.1002/jbm.a.35984 
M. Ways, T., Lau, W., \& Khutoryanskiy, V. (2018). Chitosan and Its Derivatives for Application in Mucoadhesive Drug Delivery Systems. Polymers, 10(3), 267. https://doi.org/10.3390/polym10030267

Mašek, J., Lubasová, D., Lukáč, R., Turánek-Knotigová, P., Kulich, P., Plocková, J., ... Turánek, J. (2017). Multi-layered nanofibrous mucoadhesive films for buccal and sublingual administration of drug-delivery and vaccination nanoparticles - important step towards effective mucosal vaccines. Journal of Controlled Release, 249, 183-195. https://doi.org/10.1016/j.jconrel.2016.07.036

Mazzarino, L., Coche-Guérente, L., Lemos-Senna, P. L. E., \& Borsali, R. (2014). On the Mucoadhesive Properties of Chitosan-Coated Polycaprolactone Nanoparticles Loaded with Curcumin Using Quartz Crystal Microbalance with Dissipation Monitoring. Journal of Biomedical Nanotechnology, 10(5), 787-794. https://doi.org/10.1166/jbn.2014.1768

Mendes, A., Sevilla Moreno, J., Hanif, M., E.L. Douglas, T., Chen, M., \& Chronakis, I. S. (2018). Morphological, Mechanical and Mucoadhesive Properties of Electrospun Chitosan/Phospholipid Hybrid Nanofibers. International Journal of Molecular Sciences, 19(8), 2266. https://doi.org/10.3390/ijms19082266

Nordman, H., Davies, J. R., Lindell, G., de Bolós, C., Real, F., \& Carlstedt, I. (2002). Gastric MUC5AC and MUC6 are large oligomeric mucins that differ in size, glycosylation and tissue distribution. Biochemical Journal, 364(1), 191-200. https://doi.org/10.1042/bj3640191

Ohkawa, K., Cha, D., Kim, H., Nishida, A., \& Yamamoto, H. (2004). Electrospinning of Chitosan. Macromolecular Rapid Communications, 25(18), 1600-1605. https://doi.org/10.1002/marc.200400253

Peppas, N. A., Thomas, J. B., \& McGinty, J. (2009). Molecular Aspects of Mucoadhesive Carrier Development for Drug Delivery and Improved Absorption. Journal of Biomaterials Science, Polymer Edition, 20(1), 1-20. https://doi.org/10.1163/156856208X393464

Qasim, S., Zafar, M., Najeeb, S., Khurshid, Z., Shah, A., Husain, S., \& Rehman, I. (2018). Electrospinning of Chitosan-Based Solutions for Tissue Engineering and Regenerative Medicine. International Journal of Molecular Sciences, 19(2), 407. https://doi.org/10.3390/ijms19020407

Ranc, H., Elkhyat, A., Servais, C., Mac-Mary, S., Launay, B., \& Humbert, P. (2006). Friction coefficient and wettability of oral mucosal tissue: Changes induced by a salivary layer. Colloids and Surfaces A: Physicochemical and Engineering Aspects, 276(1-3), 155-161. https://doi.org/10.1016/j.colsurfa.2005.10.033

Rathbone, M. J., Drummond, B. K., \& Tucker, I. G. (1994). The oral cavity as a site for systemic drug delivery. Advanced Drug Delivery Reviews, 13(1-2), 1-22. https://doi.org/10.1016/0169409X(94)90024-8

Samprasit, W., Kaomongkolgit, R., Sukma, M., Rojanarata, T., Ngawhirunpat, T., \& Opanasopit, P. (2015). Mucoadhesive electrospun chitosan-based nanofibre mats for dental caries prevention. Carbohydrate Polymers, 117, 933-940. https://doi.org/10.1016/j.carbpol.2014.10.026

Shenoy, S. L., Bates, W. D., Frisch, H. L., \& Wnek, G. E. (2005). Role of chain entanglements on fiber formation during electrospinning of polymer solutions: good solvent, non-specific polymer-polymer interaction limit. Polymer., 46(10), 3372-3384. 
https://doi.org/10.1016/j.polymer.2005.03.011

Sogias, I. A., Khutoryanskiy, V. V., \& Williams, A. C. (2010). Exploring the Factors Affecting the Solubility of Chitosan in Water. Macromolecular Chemistry and Physics, 211(4), 426-433. https://doi.org/10.1002/macp.200900385

Sogias, I. A., Williams, A. C., \& Khutoryanskiy, V. V. (2008). Why is Chitosan Mucoadhesive? Biomacromolecules, 9(7), 1837-1842. https://doi.org/10.1021/bm800276d

Squier, C. A., \& Kremer, M. J. (2001). Biology of Oral Mucosa and Esophagus. JNCI Monographs, 2001(29), 7-15. https://doi.org/10.1093/oxfordjournals.jncimonographs.a003443

Stie, M. B., Jones, M., Sørensen, H. O., Jacobsen, J., Chronakis, I. S., \& Nielsen, H. M. (2019). Acids 'generally recognized as safe' affect morphology and biocompatibility of electrospun chitosan/polyethylene oxide nanofibers. Carbohydrate Polymers, 215, 253-262. https://doi.org/10.1016/j.carbpol.2019.03.061

Teubl, B. J., Absenger, M., Fröhlich, E., Leitinger, G., Zimmer, A., \& Roblegg, E. (2013). The oral cavity as a biological barrier system : Design of an advanced buccal in vitro permeability model. European Journal of Pharmaceutics and Biopharmaceutics, 84(2), 386-393. https://doi.org/10.1016/j.ejpb.2012.10.021

Thirion-Delalande, C., Gervais, F., Fisch, C., Cuiné, J., Baron-Bodo, V., Moingeon, P., \& Mascarell, L. (2017). Comparative analysis of the oral mucosae from rodents and non-rodents: Application to the nonclinical evaluation of sublingual immunotherapy products. PLOS ONE, 12(9), 1-16. https://doi.org/10.1371/journal.pone.0183398

Wan, F., Nylander, T., Klodzinska, S. N., Foged, C., Yang, M., Baldursdottir, S. G., \& M. Nielsen, H. (2018). Lipid Shell-Enveloped Polymeric Nanoparticles with High Integrity of Lipid Shells Improve Mucus Penetration and Interaction with Cystic Fibrosis-Related Bacterial Biofilms. ACS Applied Materials \& Interfaces, 10(13), 10678-10687. https://doi.org/10.1021/acsami.7b19762 